\title{
Il filosofo di campagna goldoniano tradotto da Ramón de la Cruz Note di lingua e riscrittura*
}

\author{
Elena E. Marcello \\ Universidad de Castilla-La Mancha \\ elena.marcello@uclm.es
}

\begin{abstract}
Il saggio analizza dal punto di vista linguistico-letterario alcuni passi della zarzuela di Ramón de la Cruz El filósofo aldeano, traduzione-adattamento di un dramma giocoso goldoniano, allo scopo di identificare le linee maestre seguite dal drammaturgo spagnolo per la sua rielaborazione drammatica. La campionatura dei testi, che tiene conto sia delle tipologie testuali che della caratterizzazione comica o seria dei personaggi, permette di enucleare la tendenza di Ramón de la Cruz ad accentuare il carattere popolare - con un abbassamento stilistico-linguistico del dettato - delle figure comiche. Se nel caso di Don Alfonso e dello scrivano, lo scopo è quello parodistico, caricaturale; nel caso del contadino-filosofo (e della servetta, qui vista di sfuggita), gli interventi vanno, invece, in direzione opposta.
\end{abstract}

Parole chiavi: Goldoni; Ramón de la Cruz; dramma giocoso; zarzuela; traduzione; riscrittura.

\begin{abstract}
This essay discusses, from a linguistic-literary viewpoint, some movements of the zarzuela El Filósofo Aldeano by Ramón de la Cruz (which is a translation-adaptation of a goldonian "dramma giocoso"), with the aim of identifying the framework followed by the Spanish playwright in order to achieve his dramatic re-elaboration. Our sampling of the texts, which takes into account the textual typologies as much as the characterization (serious or comic) of the characters, allows us to elucidate de la Cruz's tendency to emphasise the popular character of the comic figures (which also involves a stylistic lowering of the dictation). If in the case of Don Alfonso and of the scrivener the purpose is parodic or caricaturesque, in the case of the peasant-philosopher (and of the servant, seen here in passing) the interventions work, on the contrary, in the opposite direction.
\end{abstract}

Keywords: Goldoni; Ramón de la Cruz; dramma giocoso; zarzuela; translation; rewriting.

* Quest'articolo s’inquadra nei Programmi de Ricerca ministeriali spagnoli FFI2009-07293: Goldoni en España e FFI2011-13321: Los drammi giocosi de Goldoni en la Peninsula Ibérica. 
Negli anni Sessanta del Settecento Ramón de la Cruz scrive la zarzuela El filósofo aldeano, traduzione-adattamento de Il filosofo di campagna di Goldoni. E un periodo di confronto con il teatro europeo, particolarmente denso di spunti creativi. Pur trattandosi di un confronto dettato da esigenze di botteghino e da contingenze esterne, l'operazione di adattamento dei libretti d'opera (sia sul versante comico sia su quello serio) non solo arricchisce l'esperienza teatrale dello spagnolo ma favorisce il consolidamento del genere della zarzuela. Sorvolo sui riferimenti storico-culturali e testuali dei due libretti, di cui ho trattato in precedenza e a cui rimando ${ }^{1}$, per soffermarmi in questa sede sugli aspetti più propriamente linguistici e stilistici della versione castigliana rispetto al modello italiano. Ramón de la Cruz, che rimane abbastanza fedele allo sviluppo diegetico del dramma giocoso goldoniano, adegua il testo italiano alle dimensioni (da tre a due atti) del genere musicale spagnolo, alla versificazione, al linguaggio e alle metafore della drammaturgia iberica. Qui di seguito, si analizzano alcune scelte operate dal traduttore, individuando, ove possibile, i propositi del drammaturgo. Il corpus selezionato riguarda sei brani, estratti dal manoscritto della Biblioteca Histórica Municipal di Madrid (Ms T-187-42. A) e confrontati con l'antigrafo utilizzato dall'autore (Barcelona, P. Campins, 1758), disponibile attraverso il sito www.variantiallopera.it. La scelta dei campioni tiene conto sia della varietà drammatico-testuale (arie vs recitativi; dialogo $v s$ monologo) sia della tipologia dei personaggi (comici vs seri), ai quali di solito è sotteso un determinato linguaggio. Tra i primi spiccano, da un lato, il vecchio Don Tritemio accompagnato dalla figura femminile «bassa», la servetta; dall'altro, il ricco campagnolo, la cui "filosofia» viene messa in evidenza nel titolo dell'opera. Fanno da controcanto, gli estratti riguardanti il personaggio serio, l'innamorato. Le sequenze sono le seguenti: a) frammento del duetto iniziale (I, 2) tra Don Tritemio e Lesbina, con il conseguente b) commento (I, 3) di Don Tritemio, c) il monologo (I, 5) di Nardo; d) un estratto del dialogo (II, 4) tra Don Tritemio ed il nobile Rinaldo accompagnato dal notaio; e per concludere, due pezzi chiusi e) la pena (I, 4) e f) lo sdegno dell'innamorato (II, 7).

Prima di addentrarsi nello studio della zarzuela spagnola sono di rigore alcune generalità, spesso tra loro intersecanti, che riguardano la lingua, la versificazione e la musica sia del testo di partenza (Goldoni) sia di quello di arrivo (Ramón de la Cruz). Folena ${ }^{2}$ ha dedicato pagine illuminanti alla «lingua teatrale» di Goldoni, lingua di comunicazione vicina al parlato ma non per questo

1. Cfr. Elena E. Marcello, «Da Goldoni a Ramón de la Cruz. Il filosofo di campagna a Madrid nella seconda metà del Settecento", Rivista di Letteratura Teatrale, 5, 2012, pp. 77-88. Intervento presentato al Convegno Drammi giocosi nella penisola iberica (1750-1812), organizzato a Barcellona da Teatresit-Universidad Autónoma de Barcelona dal 24 al 26 novembre 2011.

2. Gianfranco Folena, «Goldoni librettista comico», in ID., Litaliano in Europa. Esperienze linguistiche del Settecento, Torino, Einaudi, 1983, pp. 307-324; ma si vedano anche nello stesso volume, «L'esperienza linguistica di Goldoni», pp. 89-132; «Il linguaggio di Goldoni: dall'improvviso al concertato", pp. 133-160. 
"parlata» ${ }^{3}$, e in particolare, a quella dei libretti, scritti spesso di getto e in tempi brevi per soddisfare l'impellente richiesta di nuovi testi. Vale la pena riportare un'osservazione dello storico della lingua italiana sul Goldoni librettista.

Fatta la debita tara sulla differenza di peso letterario, in campo melodrammatico il Goldoni si presenta in tutti i sensi, anche linguisticamente (precarietà, contingenza, documentarietà della parola contro assolutezza e rarefazione), come un anti-Metastasio, proprio per il suo spirito di compromesso, per il suo stare alle occasioni, e perché tra tutti i librettisti europei egli è stato probabilmente il più fecondo e congeniale creatore di testi d'opera buffa, giocosa o comica, e di commedia musicale. ${ }^{4}$

Il forte legame di Goldoni con la tradizione teatrale «plurilinguistica» nostrana porta poi alla ribalta la questione dell'esportabilità delle sue opere in dialetto veneziano e di quelle in cui fanno capolino le altre «lingue» della penisola. Sul tema c'è ancora molto da indagare, ma i pochi dati a disposizione sembrano indicare la scarsa diffusione di queste pièces in Spagna. Ramón de la Cruz, per esempio, non le prende a modello delle sue riscritture teatrali. I motivi non sono forse da attribuire solo alla difficoltà linguistica, ma anche al fatto che il plurilinguismo non sia al centro dell'interesse del drammaturgo spagnolo. Resta da ricordare, seppur incidentalmente, il fatto che la lingua, nei drammi musicali, si frange tra recitativi e $\operatorname{arie}^{5}$. E se i primi tendono all'andamento prosastico, con minime inversioni dettate da esigenze di rima o verso, per i pezzi chiusi vige la tendenza contraria. Il tutto è poi strettamente connesso alla versificazione adottata dall'opera buffa ${ }^{6}$, che, a quest'altezza, ammette ancora un ampio margine di libertà. Ricapitolando, le circostanze della composizione, le necessità di comunicazione, le finalità drammatiche, la metrica e la musica sono i fattori che determinano il tessuto linguistico del testo italiano.

3. Ricorda, infatti, l'illustre linguista che Goldoni costituisce un punto cruciale della storia della lingua italiana proprio per la creazione di una lingua teatrale adatta a più di un pubblico geo-linguisticamente definito.

4. G. FolenA, "Goldoni librettista comico», op. cit., p. 309. Sul Goldoni librettista, cfr. anche Ted Emery, Goldoni as librettist. Theatrical reform and the drammi giocosi per musica, New York - Bern - Frankfurt am Main — Paris, Peter Lang, 1991.

5. Sulla specificità del linguaggio del dramma per musica, cfr. Renato Di Benedetto, «Il Settecento e l'Ottocento", in Letteratura italiana, a c. di A. Asor Rosa, vol. VI, Teatro, musica e tradizione dei classici, Torino, Einaudi, 1986, pp. 365-401; Ilaria Bonomi, «La lingua dell'opera comica del Settecento: Goldoni e Da Ponte», in Storia della lingua italiana e storia della musica. Italiano e musica nel melodramma e nella canzone. Atti del IV Convegno ASLI, a cura di E. Tonani, Firenze, Franco Cesati, 2005, pp. 49-74; Ilaria Bonomi - Edoardo Buroni, Il magnifico parassita. Librettisti, libretti e lingua poetica nella storia dell'opera italiana, Milano, Franco Angeli, 2010.

6. Sul tema si veda Paolo FABBRI, «Istituti metrici e formali», in Storia dell'opera italiana, a cura di Lorenzo Bianconi e Giorgio Pestelli, Parte II/ I Sistemi 6 Teorie e tecniche Immagini e fantasmi, Torino, EDT/MUSICA, 1988, pp. 163-233; Paolo FABBri, Metro e canto nell'opera italiana, Torino, EDT, 2007. 
Resta, pertanto, da chiedersi in che modo si sia comportato Ramón de la Cruz nei confronti della lingua e dello stile de Il filosofo di campagna. Ha adottato soluzioni linguistiche analoghe a quelle di Goldoni? Ha mantenuto le strutture metriche dell'italiano, conservando il contrasto tra pezzi cantati e recitativi? E così via. L'analisi della zarzuela El filósofo aldeano sembra confermare la tendenza, già avvertita nel teatro spagnolo coevo e in altre produzioni di Ramón de la Cruz, a ridurre i recitativi (mal sopportati in Spagna) a favore dei recitati, mentre nei pezzi chiusi, Ramón de la Cruz sembra avere, per contingenze versificatorie e meliche, un atteggiamento alquanto conservatore. Per quanto concerne la metrica, mancano studi esaustivi sulle forme usate nella configurazione delle zarzuelas o dei drammi per musica di derivazione europea. Di fatto sono poche le edizioni moderne ${ }^{7}$ che offrano un quadro delle soluzioni metriche dello spagnolo. Per di più, il presente contributo non si fonda su un'edizione critica de El filósofo aldeano che offra al lettore, dopo un'attenta collatio e constitutio textus, un testo affidabile dal punto di vista ecdotico. Ci si basa su un manoscritto conservato nella Biblioteca Histórica Municipal di Madrid, il cui confronto con altri testimoni, anch'essi manoscritti, ha già evidenziato qualche lettura divergente. Anche se si è ancora lontani da risultati concludenti sulla struttura metrica della zarzuela di Ramón de la Cruz, si profilano già alcune scelte costanti, spiegabili facendo ricorso alla tradizione teatrale spagnola dei Secoli d'Oro. In primo luogo, l'uso del romance in ottonari, metro dei parlamentos (dialoghi o monologhi informativi, descrittivi, soliloqui, ecc.), che viene applicato ai recitati, a cui è affidata l'azione. In secondo luogo, la più ampia varietà metrica dei pezzi chiusi, in cui è maggiore l'aderenza (al computo sillabico, se non alle rime) al dettato dell'italiano. Una spiegazione dell'atteggiamento conservatore di Ramón de la Cruz nei riguardi delle arie è da ricercarsi sia nell'ordito musicale sia nel fatto che, nell'andamento drammaturgico dell'opera, esse rappresentano un momento di stasi, la pausa per l'esteriorizzazione dei sentimenti. Purtuttavia, il nesso parola-musica permette ancora delle libertà al poeta drammatico. E di fatto, Ramón de la Cruz non solo depenna molti pezzi chiusi della fonte, ma richiede al compositore l'arrangiamento musicale per nuove arie e inserisce pezzi cantati tratti dalla tradizione melica spagnola (coplas, canciones, tonadillas, ecc.).

Tali premesse rendono conto sia del complesso di fattori da considerare nell'analisi della traduzione-riscrittura di Ramón de la Cruz sia della provvisorietà dei risultati. Vediamo al dettaglio i brani estratti da El filósofo aldeano.

7. Gonzalo Corona Marzol, Ramón de la Cruz, "El peregrino en su patria» (Zarzuela en dos actos). Edición, prólogo y notas..., in Anales del Instituto de Estudios Madrileños, 27, 1989, pp. 513-550; Ramón de la Cruz, Clementina, introduzione di Maria Grazia Profeti e Miguel Ângel Marín, testo critico, traduzione e note di Nicoletta Lepri, Firenze, Alinea, 2003; Ramón de la Cruz, Aquiles en Sciro, edizione di Andrea Baldissera, Como-Pavia, Ibis, 2007. Ai riferimenti sopra citati aggiungo, anche non ancora reperita, l'edizione di Ramón de la Cruz, Los portentosos efectos de la naturaleza, Edizione e studio introduttivo di Daniela Pierucci, Pisa, Felici Editore, 2008. 


\section{a) Duetto iniziale tra Tritemio e Lesbina}

La sequenza è famosa non solo per il duetto sostenuto da Don Tritemio (basso) e da Lesbina (soprano), ma anche per il contrasto creato tra le metafore elevate d'apertura (il gelsomino) e quelle degli ortaggi comuni (il «ravanello», la "cicoria» e, per finire, «l'insalata», che qui non viene riportata) che sostengono i tre momenti musicali della servetta. Queste immagini, irriverente ammiccamento sulle capacità amatorie dei vecchi rispetto a quelle dei giovani, vengono tradotte quasi letteralmente da Ramón de la Cruz, il quale, con ogni probabilità, doveva apprezzarne la vena popolare. È da notare, tuttavia, la amplificatio del dialogo tra i due personaggi, vòlta a rimarcare l'ambientazione spagnola - si ricordano che i prodotti della terra vengono raccolti e venduti sui mercati di Toledo e Madrid - e a creare nuovi giochi lessicali — quello tra il «sale» sulle verdure e la radice del verbo «salir» («sal-gan») —. D’altro canto, Ramón de la Cruz si discosta dalla fonte in determinati momenti, forse per incomprensioni linguistiche o perché mosso da una differente intenzionalità stilistica. Si prenda la voce "cantuzzare», attestata solo nell'Ottocento dalla $5^{\circ}$ edizione del Vocabolario dell'Accademia della Crusca e dal Tommaseo, e considerata "formata per ischerzo; lo stesso che Canticchiare» ${ }^{8}$. Questa voce si risolve nella metafora mitologica della «Filomena", viva in entrambi i paesi, come se Ramón de la Cruz, compreso il significato del verbo, avesse preferito eludere la patina burlesca adottando un'immagine, quella dell'usignolo, letteraria, sì, ma già cristallizzata nel Diccionario de Autoridades del $1780^{\circ}$. In direzione opposta vanno i tre interventi seguenti: nel primo, il tono sobrio con cui Lesbina nega di aver cantato canzoni d'amore ("Oh non signore») slitta nel testo spagnolo verso il registro colloquiale, con il modo di dire «no pasa acá esa moneda»; nel secondo, il «tra sé» basato sulla derivatio in rima dell'italiano "proposito: sproposito" viene risolto in modo più prosaico dal commento, sempre tra sé, in cui Lesbina esprime l'intenzione di cantare una "copla» qualsiasi per ingannare il vecchio spasimante; nel terzo, per finire, l'apprezzamento di Don Alfonso sulla vivacità della giovane - in Goldoni riassunto nell'esclamazione "Che ragazza!» — si esplicita nell'aggettivo familiare "pizpireta», ergo vivace, birichina. Più neutro sembra l'intervento riguardante la formula «scaccia questa canzon dalla memoria», con cui Don Tritemo intima alla serva di non ricordargli la vecchiaia. In spagnolo, infatti, l'espressione «el sonecillo no es malo,/ pero no vale la letra» (la musica è bella ma le parole sono sbagliate), pur mantenendo il tono familiare, appare meno categorico.

Le osservazioni sopra riportate devono poi essere connesse con l'aspetto versificatorio, qui alquanto stravolto da Ramón de la Cruz. L'alternanza tra settenari ed endecasillabi dell'italiano viene sostituita dagli ottonari assonanzati del romance e le frequenti rime baciate - che, in certo qual modo, davano

8. Consultati online: www.lessicografia.it/cruscle/ricerca.jsp e www.dizionario.org.

9. Cfr. www.rae.es. 
ritmo al recitativo (ormai trasformato in recitato) - vengono eluse. Aumentato il divario tra il recitato e il canto, Ramón de la Cruz rispetta, invece, sia la struttura che le rime delle due arie $4+4$ in quinari (abbc' abbc') e in senari (aabc' aabc').

Don Tritemio

Che si fa, signorina?

LESBINA

Un po' d'insalatina

raccogliere volea pel desinare.
Don Alfonso.

¿Y qué se hace, señorita?

JACINTA

Poco ha que bajé a la huerta

a coger una ensalada

para poner a la mesa

al mediodía.

Alfonso

¿Y la gente?

JACINTA

Luego que cargó las cestas con la fruta se marchó.

Como acostumbra a venderla

a Toledo y a Madrid...

Alfonso

Bien está ${ }^{10} \cdot \sum^{Y}$ de qué hierbas has de hacer la ensaladita?

JACINTA

¡Qué sé yo! De las primeras que salgan.

Alfonso

Ya tendrán sal.

JACINTA

Sí, señor, si se la echan.

Don Tritemio

Alfonso

Poco fa v'ho sentito a cantuzzare.

LesBina

È ver, colla padrona

mi divertiva un poco.
Pues poco ha, parece que cantaban las filomenas.

JACINTA

Cierto es, que yo y mi señora nos divertíamos a medias.

10. Si corregge il testo, ipermetro nel manoscritto («Muy bien está. ¿`Y de qué hierbas...»). 
Don Tritemio

che cantate s'avranno

E mi figuro

canzonette d'amor.

LESBINA

Oh non signore;

di questo o di quel fiore, di questo o di quel frutto,

si cantavan le lodi.

Don Tritemio

Il crederò?

LESBINA

Le volete sentir?

Don TRITEMIO

Le sentirò!

LESBINA

(Qualche stroffetta canterò a proposito) (Da sé)

Don Tritemio

(Oh ragazza!... Farei uno sproposito) (Da sé)

Lesbina

Sentite, padron bello,

la canzonetta sopra il ravanello.

Quando son giovine, son fresco e bello, son tenerello,

di buon sapor.

Ma quando invecchio

gettato sono;

non son più buono

col pizzicor.

Don Tritemio

Scaccia questa canzon dalla memoria.
Alfonso

¿Serían canciones de amor?

\section{JACINTA}

No pasa acá esa moneda; de estas o las otras flores, de estas frutas o de aquellas la alabanza se cantaba.

Alfonso

¿Y lo creeré?

JACINTA Para prueba

cantaré una, si gustáis.

Alfonso

Vaya muy enhorabuena.

JACINTA

(Procuraré asegurarle Aparte. con una copla cualquiera.)

Alfonso

(Jamás he tenido en casa Aparte. criada más pizpereta.)

JACINTA

Oíd sobre el rabanito una canción muy discreta.

Soy, cuando nuevo, ${ }^{11}$ fresco y bonito, soy tiernecito, de buen sabor;

mas, si endurezco, soy arrojado y despreciado por el picor.

\section{Alfonso}

El sonecillo no es malo, pero no vale la letra.

11. Da notare che nel manoscritto viene scritto in margine un altro brano, poi cassato e ora illeggibile. 
LESBINA

Una le vuo' cantar su la cicoria.

Son fresca e son bella cicoria novella, mangiatemi presto; coglietemi su.

Se resto nel prato, radichio invecchiato nessuno si degna raccogliermi più.

\section{Don Tritemio}

Senti, ragazza mia, questa canzone ha un poco d'allegria. Tu sei, Lesbina bella, cicorietta novella; prima che ad invecchiar ti veda il fato, esser colta dovresti in mezzo al prato.
JACINTA

Vaya sobre la achicoria otra que es mucho más fresca.

Soy fresca y hermosa, chicoria gustosa, cogedme al instante que llegue a nacer;

si en el prado crezco, presto me envejezco, $\mathrm{y}$ luego ninguno me quiere comer.

\section{Alfonso}

Esta canción sí que viene al caso y está bien puesta, y tú eres, Jacinta mía, una chicoria perfecta.

JACINTA

Sí, pero seré chicoria amarga cuando lo sea.

Alfonso

A mí me parece que, antes que te envejecieras, convendría te cogiese quien...

JACINTA
Ese asunto se queda
a mi cuidado. $[\ldots]^{12}$

\section{b) Commento di Don Tritemio}

Frammento esemplare della tendenza di Ramón de la Cruz ad abbassare il registro stilistico-linguistico del dramma giocoso goldoniano e a sfrondarne, ove possibile, l'accompagnamento musicale. Tritemio, personaggio buffo, è appena stato maliziosamente snobbato da Lesbina. Se ne è reso conto, ma persevera nei suoi intenti, convinto che potrà, con un abile corteggiamento (le lusinghe e le finezze), conquistare la servetta. Deve però prima liberarsi della figlia, concedendola in mano a Nardo che, anche se "villano", possiede ricchezza e saggezza. L'aria goldoniana è sorretta da una strofa di dieci versi 
endecasillabi e settenari con schema aAbc'dD'eFGG e sostenuta da una combinazione di lessico letterario (l'avverbio d'apertura, i verbi «lusingare» o "premere», il sostantivo "finezze») e di lingua comune (gli accrescitivi in rima, l'espressione «buon partito»), il tutto scandito dal ritmo della sporadica rima baciata sia piana («allegoricamente: niente»; «riccone: sapientone») che tronca («supererò: farò»). Il testo spagnolo è, invece, in ottonari (romance e-a). Si è, quindi, di fronte a un brano recitato. Questa saliente differenza spiega anche l'amplificatio (e degradatio) stilistica promossa da Ramón de la Cruz, che, pur mantenendo il senso generale del discorso (restano le «finezas» che, accanto ai giorni, riassumono le intenzioni del vegliardo corteggiatore), lo infioretta di espressioni colloquiali e perfino grossolane. Si inizia con l'esclamazione «demontre», «diavolo» (attestata nel Corde ${ }^{13}$ con esempi tratti proprio da questo drammaturgo, mentre i dizionari l'accolgono nel 1884) cui si abbina la coppia sinonimica "puerca cochina", che diventerà presto espressione lessicalizzata. Seguono i modi di dire "en mis barbas», "(dire le cose) in faccia», e «hacerse de pencas», ovvero, «fare la difficile» ${ }^{14}$ (perché le «pencas» pungono), e ancora l'attributo spregiativo "culiparda", qui sostantivo, testimoniato nel Corde da una frase del 1844 (di Braulio Fox: «sebosa, mermosa, asquerosa, ojisucia, podrida, culiparda, hedionda, picuda...»).

Si giunge così all'accenno alla ricchezza di Nardo che, proprio per questo, è un buon partito. In Goldoni, Don Tritemio concludeva sbrigativamente il discorso dicendo «Nardo per lei sarà: ricco, riccone/ un villano, egli è ver, ma sapientone». Una sentenza, marcata dall'effetto della rima, che univa la praticità all'interesse. Ramón de la Cruz, invece, si sofferma prima sull'unione dei beni, graficamente visualizzata dal verbo «empalmar» (Diccionario de Autoridades, 1732), che queste nozze auspiciano, e successivamente, sulla mancanza di nobiltà di Juan Bueno che viene giudicata irrilevante ("qué me importa que no tenga/ fueros de noble»). Il discorso, pedestre e materialistico, sembra degradare il personaggio di Don Alfonso. Gli attribuisce, infatti, un pragmatismo sgarbato e villano e lo priva di quella bonarietà che lo rendeva un personaggio con le sue fissazioni, buffo, ma "rassicurante», positivo. Invece, sia la morale dell'interesse che le brame amatorie trasformano Don Alfonso in una figura quasi grottesca. D'altro canto, l'assenza dei privilegi nobiliari di Juan Bueno viene compensata sia dalle sue capacità «imprenditoriali» — è una persona industriosa («hábil») — sia dai suoi possedimenti. Questi brevi commenti sul filosofo di campagna delineano un'immagine abbastanza positiva del contadino. La descrizione, infatti, è lontana dal cliché dello zotico o dell'arricchito e verrà confermata poco dopo dall'ingresso in scena di Juan Bueno.

13. Consultato on line: www.rae.es. Data: maggio 2012.

14. Attestato quest'ultimo fin dal 1665 (Corde). 
TRITEMIO

Allegoricamente

m'ha detto che con lei non farò niente.

Eppure io mi lusingo

che a forza di finezze

tutto supererò,

che col tempo con lei tutto farò.

Per or d'Eugenia mia

liberarmi mi preme. Un buon partito

Nardo per lei sarà: ricco, riccone;

un villano, egli è ver, ma sapientone.
Alfonso.

¡Esto es decir que no quiere, el demontre de la puerca cochina! ¡Y a mí, en mis barbas!

¿Cuándo lo pensaba ella, la culiparda...? ¿Mas no puede ser que yo entendiera mal o que, al verse querida, se me quiera hacer de pencas? Dejémoslo en este estado, que esto, días y finezas lo allanan. Pensemos ahora en empalmar las haciendas de Juan Bueno con las mías. ¿Qué me importa que no tenga fueros de noble, si es hábil y el más rico de esta tierra? ${ }^{15}$

\section{c) Il monologo di Nardo. Gli antenati del contadino}

Frammento relativo alla prima apparizione sul palcoscenico del filosofo di campagna, significativa sia a livello musicale che drammaturgico. In un ambiente rustico, il ricco contadinotto, vanga in mano, intona un canto al sano lavoro campagnolo e alla ricompensa che ne verrà: una sollazzevole mangiata allietata dal buon vino. È un inno al duro lavoro e al piacere delle cose semplici. Questa prima impressione viene poi precisata nel monologo, in cui Nardo, qual re di un pacifico regno, ripassa la propria genealogia. Del discorso d'apertura, colpisce anzitutto il disdegno per quelle generazioni cittadine che aspirano ad assurde ascese sociali e che, con il loro comportamento, provocano la rovina economica di intere famiglie. In campagna, invece, dove simili ambizioni non esistono, gli uomini sono integri e «sempre gl'istessi». Vi è in Nardo una consapevolezza delle proprie origini, non rinnegate né mascherate, e la volontà di sposare la vita di natura. Di questa sua filosofia, retta dall'uso della ragione, si avranno prove evidenti nel corso dell'azione scenica. Sarà, infatti, proprio lui a superare vecchi pregiudizi e, adeguandosi alle circostanze, a sposare la servetta Lesbina, rendendola, da serva, padrona. E sarà ancora lui a favorire l'amore dei due innamorati (ruolo questo enfatizzato nella zarzuela) e ad eludere le venali aspirazioni del vecchio Don Tritemio/ Don Alfonso.

La polemica sociale goldoniana viene assimilata da Ramón de la Cruz e iscritta nel tradizionale «menosprecio de corte y alabanza de aldea». Dopo il coro di labradores, il discorso di Juan Bueno appare nei contenuti molto simile a quello di Nardo, anche se risolto, dal punto di vista formale, in un romance e-a 
che sostituisce la strofa in settenari ed endecasillabi (si è pertanto davanti ad un altro recitativo trasformato in recitato). Da notare, però, l'arricchimento lessicale che va di pari passo con la reiterazione del messaggio «sociale». Mantenuta la metafora goldoniana del regno, in cui i sovrani sono gli antenati e i sudditi la zucca e il cavolo, Ramón de la Cruz procede per accumulazione, ampliando il campo ad altri frutti della terra e agli animali («...siendo sus vasallos/ granos, olivas y cepas,/ cerdos, palomas y gallinas,/ los frutales y las hierbas»). L'accenno alla pace di tale regno («sin guerras», «en paz»), che nel testo goldoniano scaturiva dal confronto con le ambizioni cittadine, si dilata nell'evocazione del vecchio focolare contadino. Lo scenario si incrementa di dettagli: dalla vecchia casa distrutta e abbandonata ( lla «deshecha/ casa, palacio innocente») in cui, seppur poveri, sono vissuti e morti in pace genitori e nonni, si passa all'immagine dell'agreste famiglia, circondata dai frutti del proprio lavoro e dai figli; un idillico quadretto a cui è sotteso un insegnamento di carattere morale, visibile in quel riferimento al peccato di superbia, che la famiglia di Juan Bueno è riuscita ad evitare "para mantenerse limpia». Pur conservando la contrapposizione goldoniana tra città e campagna, Ramón de la Cruz prolunga l'ammaestramento morale con le immagini che sorreggono l'orgoglioso giuramento del contadino. Nella clausola finale, il piacere frivolo «delle feste e dei teatri» della vita di città è sostituito dall'avido desiderio di beni terreni («los imperios de la tierra»), mentre gli oggetti della vita contadina («zappe, trebbie, rastrei, vanghe ed aratri») scompaiono, assimilati in una sintetica invocazione alla vita «de placer llena».

Vanga mia benedetta,

mio diletto conforto e mio sostegno,

tu sei lo scettro e questi campi il regno.

Quivi regnò mio padre,

l'avolo ed il bisavolo ed il tritavolo, e fur sudditi lor la zucca, il cavolo.

Nelle città famose

ogni generazion si cambia stato.

Se il padre ha accumulato

con fatica, con arte e con periglio,

distrugge i beni suoi prodigo il figlio.

Qui, dove non ci tiene

il lusso, l'ambizion, la gola oppressi,

sono gl'uomini ognor sempre gl'istessi.

Non cambierei, lo giuro, col piacer delle feste e dei teatri

zappe, trebbie, rastrei, vanghe ed aratri.
Oh bendita azada, tú eres mi cetro, y esas amenas campiñas llenas de frutos, el reino mío. Oh deshecha casa, palacio inocente, donde reinaron sin guerras, sustos ni ambición mis padres y abuelos, cuya ascendencia, para mantenerse limpia, cuidó de no ser soberbia, aquí siendo sus vasallos granos, olivas y cepas, cerdos, palomas, gallinas, los frutales y las hierbas, vivieron, cercados de hijos y de abundancias, su mesa, y aquí murieron en paz porque vivieron con ella. Allá en las grandes ciudades no hay generación exenta de cambiar de estado: unas se abaten, otras se elevan, y cada hombre vale solo 
lo que valen sus riquezas; aquí siempre son los hombres los mismos, y jamás mezcla el interés los linajes. ¡Oh vida, de placer llena, no te trocara por todos los imperios de la tierra! ${ }^{16}$

\section{d) Dialogo tra Don Tritemio, Rinaldo ed il notaio. \\ La genealogia ed i possedimenti del nobile. \\ Tra "amplificatio" ed adeguamento culturale}

Il passo scelto a continuazione è l'adeguato contrappunto, per toni e contenuti, alla presentazione di Nardo. La scena, infatti, è quella in cui l'aristocratico Rinaldo offre le prove della propria nobiltà al padre di Eugenia, che, come si sa, pensa invece a cose concrete, e cioè, a quei soldi che, più della nobiltà, offriranno alla figlia un solido futuro. Rispetto alla fonte goldoniana, il drammaturgo spagnolo modella la scenetta in modo da valorizzare, da un lato, la morale «commerciale» di Don Alfonso e, dall'altro, gli aspetti comici dell'incontro, sia a livello di res che di verba. Nel primo caso, si moltiplicano gli interventi del pater familias, che fa da spalla alle battute del notaio, reiterando la propria ideologia. Da notare a questo proposito che proprio i versi più contundenti e "trasgressivi» del vecchio - «Eso es lo que es menester,/ que nobleza sin dinero/ sirve lo propio que un cuarto/ de rosquillas a un hambriento" - vengono cassati nel manoscritto e affiancati dalla parola "no», forse a causa di scrupoli censori. Nel secondo caso, invece, Ramón de la Cruz dà risalto al notaio, qui ormai «escribano de aldea», sfruttando, da un lato, gli spunti tradizionali offerti dalla figura del pedante e del leguleio per creare l'immagine di un sciocco pieno di sé (si pensi alla puntualizzazione sul suo ruolo di scrivano pubblico " $i Y$ de Ayuntamiento!»); dall'altro, adegua i riferimenti culturali al mondo spagnolo ed incrementa le battute di Manuel in direzione burlesca. Se l'ascendenza del nobiluomo in Goldoni risaliva al re Pipino, in Ramón de la Cruz si arricchisce con i riferimenti ad Adamo e al re Godofre, mentre il noto sovrano franco (che in un altro manoscritto viene sostituito dal più famoso re godo Wamba) cede il passo ad una battuta banale fondata sulla paronomasia del nome proprio con quello del cetriolo («Pepino»). Tutti gli interventi di Don Alfonso abbassano il tono pomposo con cui lo scrivano elenca titoli nobiliari e ricchezze, sminuendone il valore, ridotto già di per sé dalla stoltizia e sussiego di chi pronuncia il discorso. Si pensi alla desertica contea ("el condado del desierto») che dovrebbe corroborare i natali di Don Fernando oppure agli avi tanto lontani nel tempo da avere nomi in greco piuttosto che in latino!

16. Ms. T-187-42, A, 2o Apunte, della Biblioteca Histórica Municipal di Madrid, f. 9r-v. Si è corretta la lettura del $\mathrm{ms}$ «mi abuelos». 
TRitemio

Se denaro vorrà, ghe ne darò, purché sicuro sia con fondamento, e che almeno mi paghi il sei per cento. Ma che vedo? È colui che mi ha chiesto la figlia. Or che pretende? Col notaro che vuol? Che far intende?

RINALDO

Compatite, signor...

TRITEMio

La riverisco.

\section{RinALDO}

Compatite se ardisco

replicarvi l'incommodo. Temendo che non siate di me ben persuaso, ho condoto il notaro, il qual patente e chiaro di me vi mostrerà titolo, parentele e facoltà.

Tritemio

(È ridicolo invero.)

\section{Capocchio}

l'istrumento rogato

Ecco, signore,
Alfonso

$¡$ Usted parece que viene a tomar dinero a censo! Yo le daré, pero habéis de pagar quince por ciento.

\section{Fernando}

Señor, con licencia vuestra venía...

\section{Alfonso}

Ya caigo en ello. Vos sois el que esta mañana pretendía ser mi yerno, sin más ni más; digo que no quiero porque no quiero.

\section{Fernando}

Perdonadme si atrevido segunda vez os molesto, y no creyendo que pueda nacer vuestro menosprecio, mas de que ignorar quien soy, manifestaros pretendo por mano de un escribano público...

\section{Manued}

¡Y de Ayuntamiento!

FERNANDO

...mis títulos, facultades, blasones y parentescos.

Manuel

Aquí está todo más claro que la luz. Ese primero es un árbol donde, antes de Adán, prueba cien abuelos.

Alfonso

Decid los nombres. A ver si conozco algunos de ellos.

Manuel

Están todos en latín. 
d'un ricco marchesato;

ecco l'albero suo, da cui si vede che per retto camino vien l'origine sua dal re Pipino.

\section{TRITEMio}

Oh caperi! Che vedo?

Questa è una cosa bella in verità.

Ma della nobilità, signor mio caro, come andiamo dal par con il denaro?

RiNALDO

Mostrategli i poderi, mostrategli sinceri i fundamenti. (a Capocchio)

CAPOCCHIO

Questi sono istrumenti

di comprede, di censi, di livelli.

Questi sono contratti buoni e belli.
Alfonso

Creí que estaban en griego.

MANuel

Línea materna en que prueba venir por camino recto del rey Godofre.

Alfonso

$$
\text { No sé }
$$

quién es ese caballero.

Manuel

Título de baronía, a merced y privilegio del rey Pipino ${ }^{17}$.

Alfonso

$$
\text { Pepino }
$$

has de decir, majadero.

Manuel

Papeles pertenecientes

al condado del desierto.

Alfonso

Aguárdese usted, y digamos:

¿acompaña a todo eso

la riqueza?

\section{Fernando}

Se supone.

Mostradle los instrumentos

de posesiones, tributos,

raíces, casas y fueros.

\author{
Alfonso \\ Eso es lo que es menester, \\ que nobleza sin dinero \\ sirve lo propio que un cuarto \\ de rosquillas a un hambriento. \\ Vaya, señor secretario, \\ con distinción.
}

\section{Manuel}

cada cosa de por sí.

Aquí os muestro

Vos iréis reconociendo. ${ }^{18}$

17. Da notare la variante conservata nel ms della $B N E$ : «del rey Bamba, que murió sin hijos».

18. Ms. T-187-42, A, 3º Apunte, della Biblioteca Histórica Municipal di Madrid, f. 4r-v. 


\section{e) La pena di Rinaldo}

Pezzo chiuso che, nell'edizione catalana del 1758 , sostituisce l'originario «Taci amor, del seno mio» e conferma l'antigrafo usato dal drammaturgo spagnolo. Scritto per un soprano, l'aria viene assegnata in Spagna alle attrici finché il ruolo passa agli interpreti maschili. Tralasciando la questione del timbro vocalico, si è di fronte a un passo per musica intonato dalla figura seria, e quindi, connotato linguisticamente dal registro letterario, e plasmato in settenari $5+4$ con schema abcbd' efed'. Dal punto di vista metrico, Ramón de la Cruz conserva strofa, verso e rime, ma varia leggermente lo schema (ababc' deec'). Per quanto riguarda, invece, la resa linguistica, il drammaturgo spagnolo riduce la triade di termini legati al campo semantico dell'infelicità (misero-affannirigore) al binomio «infelice-afanes», mentre conserva quasi totalmente sia l'accusa contro la sorte avversa («la suerte no me quiere») sia l'aspirazione a trionfare. In quest'ultimo caso, però, preferisce mettere in risalto la fortezza dell'amante (il "pecho altivo») piuttosto che il «rigore» della sorte. Nella quartina seguente, invece, viene mantenuto l'effetto di dissimulazione ritmica, ma le lacrime, che provocano il tormento dell'innamorato, non vengono più attribuite alla donna amata ma al proprio Fernando.

No, non mi vuol sì misero, a mille affanni in seno, la sorte troppo barbara, del suo rigore almeno spero di trionfar.

Ah chi ben ama intende se sia crudel tormento, quel ciglio che m'accende vederlo lagrimar.
No, no tan infelice,

la suerte no me quiere, $\mathrm{y}$, aunque afanes predice, mi pecho altivo infiere que, al fin, ha de triunfar.

¡Ah, quien de amor entiende diga si es sentimiento, cuando de amor aliento, verme de amor llorar. ${ }^{19}$

\section{f) Lo sdegno di Rinaldo}

In Goldoni lo sfogo dell'innamorato tradito (in settenari con schema 6+6 abbacd' abbacd') viene rivolto sia alla giovane amata (qui «figlia»), sia al padre di lei che impedisce l'esito felice dell'idillio. Entrambi sono accusati di tradimento da un Rinaldo ferito e addolorato. Il testo si infittisce di aggettivi negativi. La donna viene tacciata prima d'ingratitudine («ingrata», "crudele») e poi incolpata per aver ingannato le speranze dell'amante avvivando, con una metafora di lunga tradizione lirica, il fuoco dell'amore; il genitore, invece, è accusato di crudeltà («spietato», «empio», «audace»). Nel finale il cuore offeso clama vendetta. In Ramón de la Cruz, l'aria viene ampliata e risolta nello schema, sempre in settenari, 4+7+5 abbc'deeffec' gghhc'. La prima novità riguarda gli interlocutori. L'invettiva, infatti, è rivolta, da un lato, a Leonarda e, dall'altro, alla complice Jacinta. L'amata è una traditrice ("traidora») perché 
si è presa gioco ("de mi fe te burlaste») del giovane; ha agito come tutte le donne, con volubilità, con incostanza; d'altro canto, chi ha ordito l'inganno ed il tradimento è la servetta. Il discorso si avvia sui binari della consolidata tradizione misogina e delle convenzioni teatrali che vedono i servitori abili creatori di intrighi. Con acume, Ramón de la Cruz prolunga il gioco scenico fondato sull'alternanza degli interlocutori fino alla promessa, energica ma prosaica («le sacaré al instante/ del pecho el corazón»), di una vendetta, che viene rivolta alle due donne e all'ipotetico rivale.

Perfida figlia ingrata, padre spietato indegno, non so frenar lo sdegno, l'alma si scuote irata.

Empio, crudele, audace, Pace per me non v'è. (Or all'una, or all'altro)

E tu che alimentasti sinora il foco mio colla speranza, oh Dio!, così tu m'ingannasti? L'offeso cuor aspetta vendetta, anche di te.
Dime por qué, traidora, (A Leonarda) de mi fe te burlaste, di tú por qué engañaste ( $A$ Jacinta) mi noble inclinación; mas, ¡ay!, que ya aunque tarde, veo que ambas sois mujeres.

Tú mudas pareceres sin subsistir en nada; y tú, que eres criada, haces como quien eres urdiendo la traición.

Mas ¡ea! valor mío, ¿para cuando es el brío? Desprecio tu mudanza, (a Leonarda) de ti tendré venganza, (a Jacinta) $\mathrm{y}$ a ese feliz amante le sacaré al instante del pecho el corazón. ${ }^{20}$

Questo saggio sulla traduzione-riscrittura de Il filosofo di campagna pecca, ne sono cosciente, di incompletezza. Le ipotesi proposte devono essere sottoposte a verifica attraverso un esame completo della zarzuela, tuttora priva di edizione critica, e del restante teatro per musica di Ramón de la Cruz. Tale disamina permetterebbe di individuare le variabili e le costanti del suo misurarsi con il teatro italiano ed europeo. Pur nella precarietà delle deduzioni, però, è possibile concludere che l'operazione dello spagnolo segue, rispetto ai personaggi buffi, due impulsi opposti: da un lato, tende ad accentuarne gli aspetti comici (il vecchio, il notaio); dall'altro, a valorizzarne le qualità personali (la laboriosità del contadino, l'arguzia della serva). Ovviamente, sono ancora molte le questioni irrisolte. C'è da chiedersi in che modo questi esperimenti di traduzione e riscrittura favoriscano le produzioni «originali» di Ramón de la Cruz, dai sainetes alle zarzuelas, resta da vagliare se questa predilezione per i ruoli «bassi» faciliti il loro protagonismo nel teatro breve e studiare in modo più approfondito il rapporto tra parola e musica, e così via. Purtuttavia, queste prime approssimazioni su El filósofo aldeano indicano almeno un cammino da percorrere.

20. Ms. T-187-42, A, 3º Apunte, della Biblioteca Histórica Municipal di Madrid, f. 7v. 